\title{
Mechanisms of left-right asymmetry and patterning: driver, mediator and responder
} Hiroshi Hamada ${ }^{1}$ and Patrick P. L. Tam ${ }^{2 *}$

Addresses: ${ }^{1}$ Developmental Genetics Group, Graduate School of Frontier Bioscience, Osaka University, Japan and ${ }^{2}$ Embryology Unit, Children's Medical Research Institute and Sydney Medical School, University of Sydney, New South Wales, Australia

* Corresponding author: Patrick P. L. Tam (ptam@cmri.org.au)

FI000Prime Reports 2014, 6:110 (doi:10.12703/P6-I10)

All FI000Prime Reports articles are distributed under the terms of the Creative Commons Attribution-Non Commercial License (http://creativecommons.org/licenses/by-nc/3.0/legalcode), which permits non-commercial use, distribution, and reproduction in any medium, provided the original work is properly cited.

The electronic version of this article is the complete one and can be found at: http://fl $000 . c o m / p r i m e /$ reports/b/6/IIO

\begin{abstract}
The establishment of a left-right (LR) organizer in the form of the ventral node is an absolute prerequisite for patterning the tissues on contralateral sides of the body of the mouse embryo. The experimental findings to date are consistent with a mechanistic paradigm that the laterality information, which is generated in the ventral node, elicits asymmetric molecular activity and cellular behaviour in the perinodal tissues. This information is then relayed to the cells in the lateral plate mesoderm (LPM) when the leftspecific signal is processed and translated into LR body asymmetry. Here, we reflect on our current knowledge and speculate on the following: (a) what are the requisite anatomical and functional attributes of an LR organizer, (b) what asymmetric information is emanated from this organizer, and (c) how this information is transferred across the paraxial tissue compartment and elicits a molecular response specifically in the LPM.
\end{abstract}

\section{The preamble}

Early embryogenesis proceeds by breaking the symmetry, albeit radial or planar, of the embryo architecture to generate regionalized tissue patterns along the three orthogonal body axes: the anterior-posterior (AP), dorsoventral (DV) and LR axes. In the post-implantation mouse embryo, the prospective AP axis is heralded by the polarization of the head primordium on the anterior side and the primitive streak on the posterior (tail) side of the embryo, while the prospective DV axis is delineated by the stacking order of the three germ layers: the ectoderm being dorsal, the endoderm being ventral and the mesoderm between the two. An axiom of geometry stipulates that, in a three-dimensional structure, once the orientation of two orthogonal axes is delineated, the third axis would be spatially defined by default. Whether the body axes of an embryo are established in such a manner, and which of the three is the last to be defined, is not known. It is often argued that LR is the last axis to be specified and, in terms of the expression of currently characterized molecular markers, it appears last. However, a recent study showed that scrambling the blastomeres and re-constructing the eight-cell mouse embryo could lead to an increased frequency of reversed direction of axis rotation of midgestation embryo. This finding suggests that the timing for defining LR asymmetry, in the context of specifying the spatial information pertaining to LR body patterning, might be pushed back to the pre-implantation stage of mouse development [1].

It is important to understand that, beyond specifying the orientation of the axis, additional information is required for determining its polarity. In this context, the tissue patterns in the lateral body axis are further refined by breaking the bilateral symmetry to create LR difference in the body plan. This will be translated into the asymmetry in tissue morphogenesis, such as the different rate of cell proliferation and asymmetric growth of the gut mesentery; and the situ of internal organs, such as the different anatomy of the left and right lung, lopsided positioning of organs, such as the stomach, 
liver, pancreas and spleen, and the rotation of the gut. This commentary focuses on the mechanistic events and the developmental processes that underpin the establishment of the LR body asymmetry during embryonic development.

\section{To build a LR organizer}

The structure that acts as the LR organizer of the mouse embryo is the node (Figure 1). Cells of the node are recruited from the epiblast cells in the anterior region of the primitive streak. These cells are organized into a domain of columnar epithelium (known as the ventral node) that is intercalated between the anterior notochord and the primitive streak in the midline of the embryo [2,3]. The formation of the ventral node is dependent on the activity of transcription factors (e.g. Brachyury, NOTO, forkhead box protein A2 [FOXA2], and zinc finger of the cerebellum [ZIC3]) and signaling pathway (such as Nodal and Notch) [4-10]. However, it is unclear how these activities may control the morphogenesis and the placement of the ventral node. A recent study [11] has highlighted the role of a cell-matrix interaction mediated by the activity of the integrin $\alpha 531$ receptor and its extracellular ligand, fibronectin, in shaping and positioning the node. In the Itga5-null and Fn1-null embryo, the ventral node has the pointed pole facing posteriorly (instead of anteriorly), with a shallow pit and seated off the midline. Some nodal cells are overlaid by the visceral endoderm, thus giving an impression that the node has an irregular shape. Cells of the Fn1-null ventral node, in particular, do not display the apical F-actin rings or the constriction of the apical part of the cell that faces the nodal pit. It remains unclear how the loss of integrin $\alpha 5 \beta 1$ may alter the cell-matrix

Figure I. The anatomy of the left-right organizer of the mouse embryo

\section{(a)}
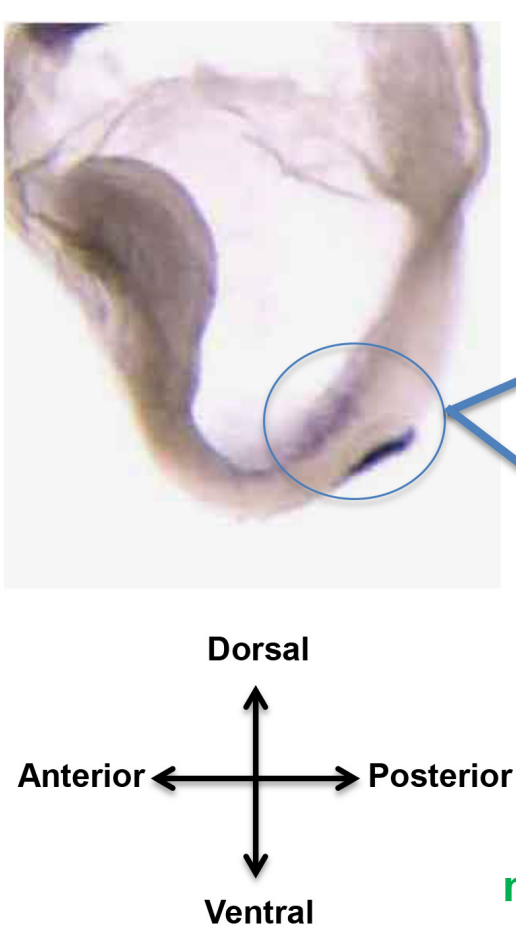

(b)

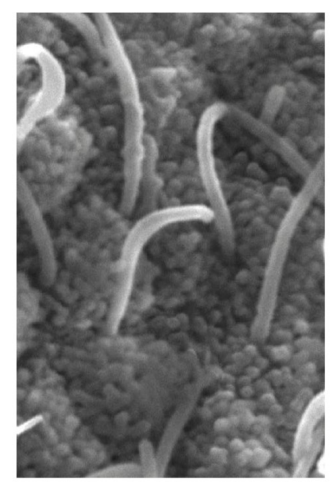

(c)

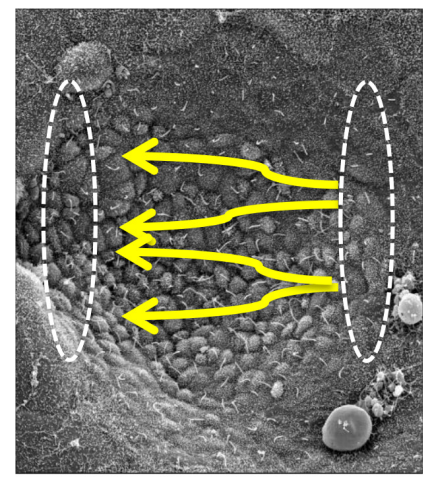

Left
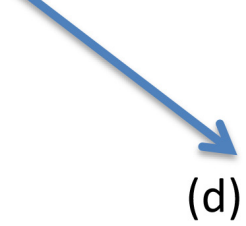

(d)

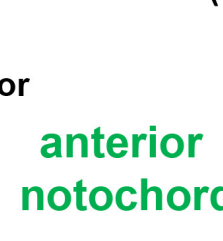

parietal yolk sac

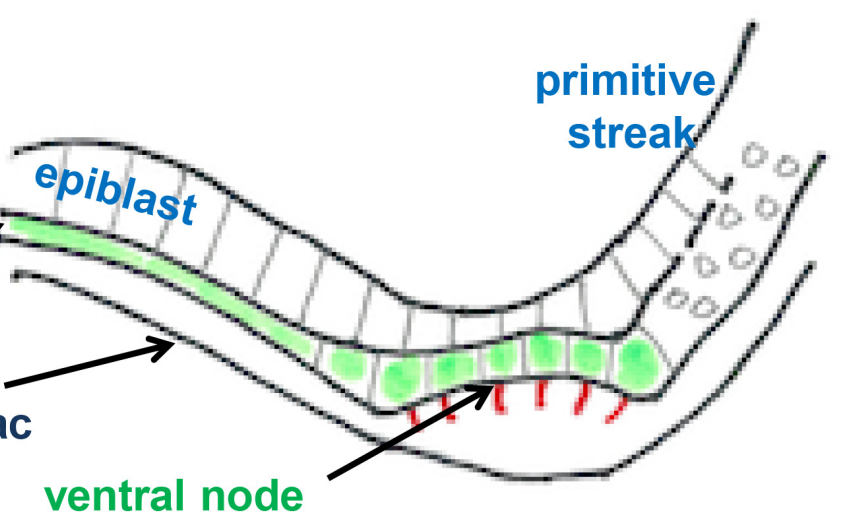

(a) The Nodal-expressing ventral node (circled) of the E8.0 early-head-fold stage embryo.

(b) The monocilia on the apical surface of the nodal pit cells.

(c) The tracing of the leftward fluid flow across the ventral node. Motile cilia are located at the central region of the node, while immotile cilia are present at the periphery of the node cavity (marked by dashed lines).

(d) The ciliated surface of the left-right (LR) organizer, which is intercalated between the anterior notochord and the primitive streak, opens to fluid-filled cavity enclosed by the ventral node and the parietal yolk sac. 
interaction in a mechanistic context to control the morphogenesis of the ventral node and the acquisition of apicobasal polarity of the nodal pit cells. Both Itga5null and Fn1-null embryo display abnormal LR patterning. These findings show that the formation of a ventral node with proper geometry, comprising cells displaying a proper apicobasal polarity at the midline, is a prerequisite of LR patterning.

Most of the cells located at the central region of the ventral node (the pit cells) of the mouse embryo are decorated with a motile cilium. The cilium is assembled from the motor protein dyneins, intraflagellar transport proteins and axonemal proteins, together with many other proteins [12] (Figure 1). The assembly of the axonemes and the cilia proper is regulated by the activity of the ciliary targeting complexes that interact with Pitchfork protein, the activation of Aurora A kinase and the deployment of FOXJ1 by NOTO to transactivate Rfx3 and other ciliary genes [13]. The basal bodies of the motile cilia are located on the posterior side of the pit cells and the cilia are tilted towards the posterior side of the node due to the dome-shape of the cells. These motile cilia display clockwise rotational movement (when viewed from the apical aspect of the cell). The posterior tilting of the motile cilia, which determines the elevation (relative to the cell surface) of the forward (high and leftward) and return (low and rightward) strokes of the cilia is instrumental in propelling the fluid across the node. This rotational action of the cilia creates a leftward flow of the fluid across the ventral node [14] (Figure 1). It has been shown that the immediate effect of the fluid flow that is initiated soon after the onset of ciliary rotation is sufficient for establishing the LR asymmetry [15]. However, it may be noted that the flow of nodal fluid in the mouse embryo is generated within a confined space enclosed by the ventral node and the parietal yolk sac (Figure 1). The dynamics of nodal flow in vivo have not been examined. It is possible that the flow pattern in this confined space may be very different from that observed in an open ventral node in an experimental setting.

The polarized location of the motile cells may be driven by the activity of the planar cell polarity pathway revealed by the different distribution of planar cell polarity factors (e.g. Prickle2, Vangl1 and Dishevelled) to the anterior and posterior sides of the ventral node cells $[13,16]$ and the activity of the Foxj1-Noto molecular cascade [17] . It is of interest that in the Itga5- and Fn1null embryo, the structure and the posterior position of the cilia on the pit cells are unaffected in the malformed ventral node. There is, however, ectopic expression of Arl13b, a ciliary protein associated with the primary cilia, on the basal side of the Fn1-null pit cells, though the functional significance of this defect is not known [11]. Whether an effective leftward nodal flow is still generated by the action of the motile cilia in the mutant embryo has not been examined. The fact that the mutant embryos display an LR patterning defect suggests that any fluid flow that may be generated is of little functional consequence.

Motile cilia also generate unidirectional fluid flow in the LR organizer of fish and frog embryos, the Kupffer's vesicle and the gastrocoel roof plate, respectively [18]. In the Kupffer's vesicle, which contains an enclosed space lined by ciliated cells, the pattern of asymmetric fluid movement (tracked by live-imaging in situ) is influenced by the length of cilia [18]. In the mouse, the length of the cilia increases progressively under the influence of Notch signaling activity following ciliogenesis [8]. In Pifo- and Noto-mutant mouse embryos, the nodal cilia are short and malformed, which may compromise the efficiency of fluid propulsion $[5,13]$.

In the Kupffer's vesicle, the spatial distribution of the ciliated cells (which are predominantly clustered in the dorsal-anterior region of the vesicle) and the activity of cilia with a high beat frequency that may generate most of the fluid flow activity are key to the generation of a productive fluid flow [19]. In this regard, the abnormal shape of the LR organizer and the re-distribution of the bulk of ciliated cells to the anterior domain of the ventral node [11] in the Itga1- and Fn1-null embryo may be contributing factors to the LR defects. However, it remains unclear if these abnormalities of the ventral node are associated with aberrant ciliary function and nodal flow.

In the zebrafish, the fluid flow generated by one or two cilia on one side of the vesicle is sufficient to determine the LR pattern of the liver and heart [19]. Similarly, in the Dpcd mutant mouse embryo as few as two motile cilia in the ventral node can fulfil the function to drive LR patterning and this attribute appears to be independent of the position of the cilia in the ventral node [20]. In the mutant embryos that only have a few cilia and still show normal LR pattern, these cilia are correctly tilted. Most likely it is the direction of the flow, not the velocity, that is important for LR symmetry breaking.

In contrast to the pit cells, the majority ( $90 \%)$ of the cells located at the edge of the node (the crown cells) have immotile cilia $[15,21]$. Diversity in the cilia type is generated by the activity of lineage-specific transcription factors on the target genes, such as polycystic kidney disease $2(P k d 2)$ and $P k d 111$. Both genes are expressed in the node $[22,23]$. They encode the $\mathrm{Ca}^{2+}$ channel and the 
putative molecule for force sensing, respectively, and are regarded as components of the mechanoreceptor on the cilia. In view of the difference in the motility, these two types of monocilia are reputed to serve different functions in the LR patterning process: with the motile cilia generating the directional fluid flow and the immotile cilia being the receiver of the morphogenetic information, for example, by sensing the direction and force of the fluid flow.

In summary, our knowledge has pinpointed the minimal anatomical and functional attributes of an LR organizer for the mouse embryo. It would be a midline epithelial structure that adopts a pit-like configuration and is exposed to a confined fluid-filled space. The central part of the epithelial pit is populated by planar polarized cells (the nodal pit cells), each carrying a motile monocilium at a posterior location on the apical aspect of the cell. The rotational activity of these cilia in a small subpopulation of strategically clustered cells can generate sufficient locomotion to drive a unidirectional flow of fluid across the organizer, with the efficacy of the flow determined by the geometry of the organizer and the enclosure. Incorporated into this organizer are cells with means to perceive the positional information conveyed by the fluid flow or other processes connected to the ciliary motion and cellular polarity.
While LR symmetry breaking in several vertebrates involves directional flow that is generated by rotational movement of cilia, there are exceptions. The chick embryo uses a different strategy for symmetry breaking: leftward movement of Hensen's node cells instead of the ciliarydriven fluid flow [24]. This leftward cell movement is transient and required for subsequent LR asymmetric gene expression in the lateral plate. A recent study [25] shows that $\mathrm{N}$-cadherin is responsible for terminating this cell movement. Furthermore, the pig embryo seems to lack motile cilia in the ventral node [24].

\section{What is the nature of the patterning information received by and emanated from the LR organiser?}

The direction of fluid flow is critical for eliciting the proper asymmetric enhancement of Nodal expression and the downstream molecular activity in the perinodal tissues. Reversing the flow across an exposed node by external means can switch on the left-specific molecular activity on the right side of the mouse embryo cultured in vitro $[26,27]$. It is, therefore, plausible that the fluid flow elicits a mechanical trigger in the responding cells, most likely the crown cells, in the peripheral region of the ventral node (Figure 2). In the mouse embryo, the pit cell-specific expression of $P k d 2$ fails to rescue LR defects of the $P k d 2^{-/-}$embryo, suggesting that motile cilia of pit

Figure 2. Morphogenetic information of the left-right organizer

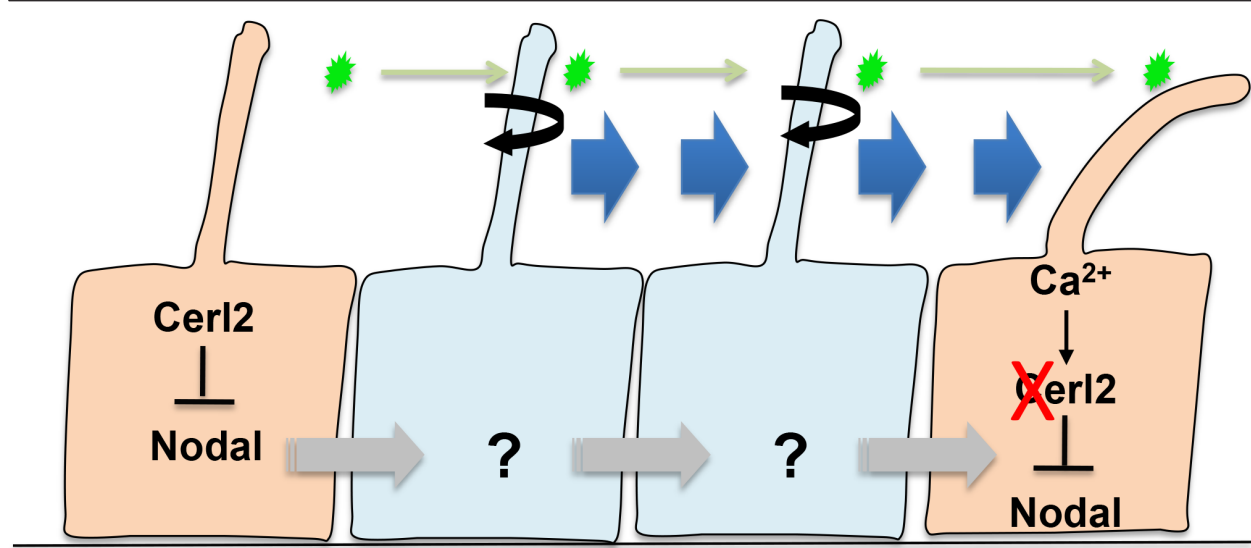

Right

\section{Left}

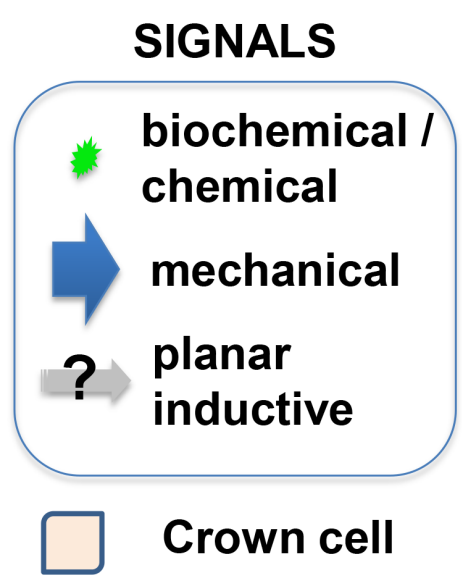

Pit cell

The rotational motion of the motile cilia of the pit cells drives the flow of the fluid to the left hand side of the left-right (LR) organizer. The mechanical force elicited by the flow of fluid may be sensed by the immotile cilia of the crown cells. The crown cell (and its immotile cilia) may also sense the biochemical factors or small chemical compounds that are transported by the fluid flow across the organizer. Whether there are other inductive/positional signals that are transferred in a planar and vectorial manner within the epithelium of the ventral node is not known. Upon sensing the information, the crown cell activates the $\mathrm{Ca}^{2+}$ ion channels mediated by the activity of the Pkd2-PkdIII complex. The channel activity is transduced via an as yet unknown mechanism that leads to the degradation of the Cerl2 mRNA and releases the repression of Nodal activity in the crown cells on the left side of the organizer. The different level of Nodal activity between the right and left side of the node creates the first left-right asymmetry downstream of the organizer activity. 
cells are unable to sense the flow [21]. However, there may be some differences between species. For example, motile cilia of the Kupffer's vesicle of the medaka embryo may sense the fluid flow because all/most of the cilia in the Kupffer's vesicle are of the motile type [22]. The presence or absence of immotile cilia in the Kupffer's vesicle needs to be investigated further.

It is not known how the fluid flow is sensed by the immotile cilia. Various parameters, such as the direction of the flow, the amplitude of the mechanical force, and the frequency of the impacting waves might be gauged. Alternatively, the immotile cilia may recognize certain as yet unknown molecules that are transported by the flow toward the sensor, such as signaling ligands, morphogenetic factors, or small chemical compounds (Figure 2). There is no direct evidence for or against either possibility. As for the latter possibility, no candidate molecule or its carrier of the appropriate physicochemical properties that could be delivered by the flow has been identified. It is also possible that the rotational cilia activity and the directional fluid flow are reflecting an underlying process of relaying planar inductive signaling or electrochemical flux activity across the epithelial cells of the ventral node. While there is compelling evidence supporting the fluid transport of small particles naturally present in the Kupffer's vesicle in the Zebrafish [19] and of lipophilic-dye-labelled membrane vesicles in ventral node of the mouse embryo in vitro [27], they do not strictly distinguish between the mechanosensory and chemosensory models. To directly address this issue, it is necessary to develop a new experimental system that could test the functional output of the fluid flow in a biochemical, biophysical and/or hydrodynamic context.

Irrespective of whether immotile cilia of crown cells may act as mechano- or chemosensors, crown cells respond to the flow, most likely via Pkd2-mediated $\mathrm{Ca}^{2+}$ signaling. The most immediate target of the flow is Cerl2 (also called Dand5), a gene encoding a Nodal antagonist, which is the mouse homologue of Coco (frog) and Charon (fish) genes $[20,28,29]$ (Figure 2). Flow-mediated signaling regulates Cerl2 expression not at the transcriptional level but at posttranscriptional level. Cerl2 mRNA seems to be degraded more rapidly on the left side of the node in response to the flow via some regulatory activity on the 3'- untranslated region (UTR) sequence [30]. The precise mechanism of Cerl2 mRNA degradation, however, remains unknown. The outcome of the differential sustenance of Cerl2 mRNA between the two sides of the LR organizer leads to a higher level of expression of Cerl2 in the crown cells, and dampens the Nodal activity on the right side of the node.

The patterning information emanating from the LR organiser is likely to be Nodal in the form of a Nodal-GDF1 heterodimer that is produced and secreted by crown cells. Nodal is expressed by the crown cells on both sides of the node but is more abundant on the left side. This molecular cascade of activity from the leftward flow to the down-regulation of Cerl2/Coco/Charon mRNA on the left side and enhancement of left side Nodal activity at the node is conserved among fishes, frogs and mice. Cerl2 protein is later translocated across the node from the right side to the left side, which renders the left-sided enhancement of Nodal activity transient [31].

\section{How is this information conveyed to the target tissues to elicit an asymmetric response?}

The target tissue of the node-derived signal is the LPM. Thus, signals from the node will reach the lateral plate and induce the asymmetric expression of Nodal on the left side. This asymmetric Nodal expression is positively regulated by Nodal itself and negatively regulated by feedback inhibitors Lefty 1 and Lefty2, making Nodal expression in LPM very dynamic and transient. Nodal signaling will induce Pitx2 expression in the left LPM. Unlike Nodal expression in LPM, asymmetric Pitx2 expression in LPM persists for a considerable period and executes LR asymmetric orga-nogenesis (reviewed in [32]).

The node-derived signal that induces asymmetric Nodal expression in LPM is most likely the Nodal-GDF1 heterodimer. Previous data suggested that this heterodimer is transported from the node to the lateral plate, presumably via the intraembryonic route (reviewed in [32]) (Figure 3). Node cells secrete vesicular parcels called nodal vesicular parcels (NVPs) [24], but it is not known if NVPs carry the signaling molecules that regulate asymmetric Nodal expression in an instructive way. The transport of the heterodimer may be facilitated by the interaction with sulfated glycosaminoglycan in the extracellular matrix along the route from the node to the LPM [33]. Alternatively, the Nodal protein that is secreted into the node cavity may be transported toward the left side of the node by the flow and may be incorporated, via the crown cells, into the endoderm. The Nodal signal may then be relayed in a planar autocrine manner across the endoderm cell layer, eventually reaching the LPM where it activates the Nodal gene locally. However, this scenario is considered unlikely for several reasons. For one, exposure of the endoderm to Nodal by incubation of mouse embryos in the culture medium supplemented with recombinant Nodal protein does not affect Nodal expression in LPM, neither unilaterally or bilaterally, nor does it induce nonLPM Nodal expression. However, injection of Nodal protein into the right side of embryos induces Nodal 
Figure 3. Transfer of left-right patterning information to the lateral plate mesoderm

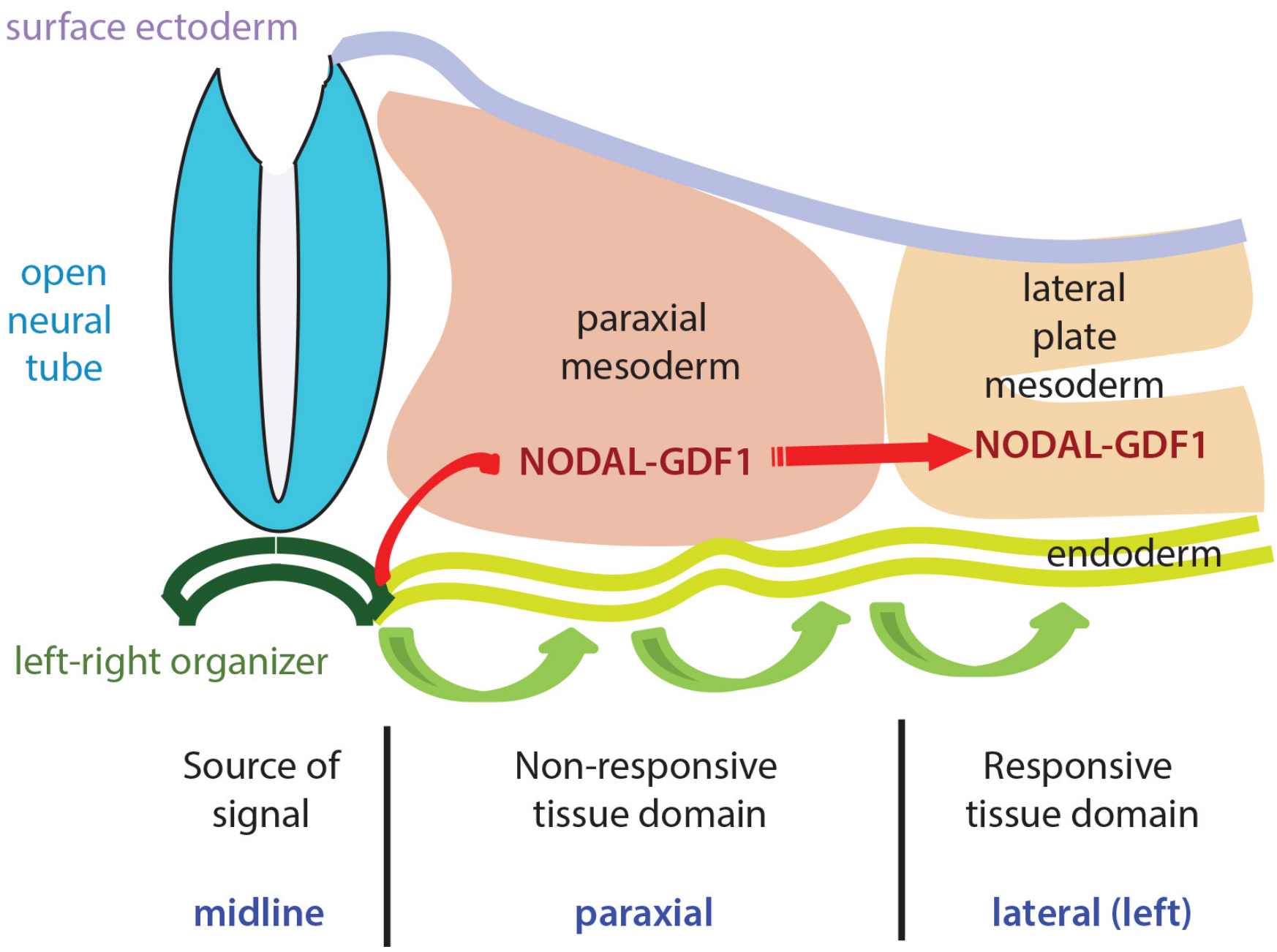

The output of left-right (LR) asymmetry information from the crown cells (and the perinodal cells) can be transmitted through the paraxial mesoderm to reach the lateral plate mesoderm. The signal is reputed to be the Nodal protein itself, or as Nodal-GDFI complex. The integrity of the gap junction of the endoderm cells has been shown to be instrumental for LR tissue patterning. In this context, it is envisaged that the LR asymmetry information may be relayed successively by cells in the endoderm from the LR organizer to the lateral plate mesoderm. It is not known how the asymmetry information conveyed through either the mesoderm or endoderm conduit can elicit a specific response in the lateral plate mesoderm but not elsewhere in the nonresponsive tissue domain.

expression on the right LPM [33]. In the LPM, repression of Nodal-induced Pitx2 expression can be attributed to the competition by bone morphogenetic protein (BMP)/ pSMAD1 signaling that limits the availability of SMAD4 for Nodal signaling. Overexpression of Smad4 in the mesoderm at the right side LPM activates ectopic Pitx2 expression that could be reversed by co-expressing a constitutively active activin receptor-like kinase 6 (ALK6) receptor [34]. In contrast, the enforced expression of either Smad4 or Alk6 in the endoderm has no effect on the asymmetric expression of Pitx2 in the LPM. It is possible that Nodal activity emanating from the crown cells activates other signaling pathways that act in a paracrine manner, either in the mesoderm or the endoderm, to transmit the Nodal induction signal to the LPM. Recent reports on Sox17 mutant mouse [35-37] suggest that gap junctional proteins in the endoderm are essential for the signal transfer. How to integrate these observations into a unified mechanism is a challenging proposition. One possibility, perhaps the simplest one, is the Viotti-Oki model [38], in which endoderm-derived signals would be required for transport of active Nodal 
protein from the node to LPM. However, alternative models may be also possible.

Sox17 mutant embryo that shows LR patterning defects [35] loses the expression of Ihh in the endoderm adjacent to the LPM, which concurrently does not express Ptch1, a target of hedgehog signaling [39]. A small GTPase, Rab23, which represses GLI activity in the hedgehog pathway, is required for normal Nodal and Lefty $1 / 2$ expression in the node, floor plate and the LPM, and loss of Rab23 is associated with defective LR asymmetry [40]. The involvement of Rab23 in LR patterning raises the possibility that a localized hedgehog signal from the endoderm may underpin the specific activation of Nodal response by the LPM. However, Rab23 function in Nodal signaling is found to be independent of Gli2 or Ptch1mediated hedgehog signaling activity [36]. This is, therefore, not consistent with a role for hedgehog activity from the endoderm in facilitating the LPM response to the LR patterning signal.

Irrespective of the mechanism and route of signal transfer, the tissue-specific response of the left LPM to the nodederived signal poses the question of why the tissue intercalated between the node and LPM, such as the paraxial and intermediate mesoderm, would not respond to the Nodal-GDF1 complex as it traverses through the mesoderm or the endoderm conduit. When Nodal or Pitx2 is induced ectopically on the right hand side of the embryo, the expression domain is invariably confined to the LPM as in the proper left hand side $[23,28,30,33,35]$. This may point to the existence of a zone of non-responsiveness on both sides of the embryo, which ensures that only the LPM is targeted by the LR patterning signal from the node (Figure 3). Such non-responsiveness to Nodal may be due to the absence of both Cryptic and FoxH1 from the tissue. It is plausible that LPM-specific response is instigated by the presence of antagonists, for example, Lefty, which is reputed to have a different "diffusivity" from Nodal [41]. In addition, the absence of agonist and transcription regulators (such as FoxH1) in the paraxial mesoderm may disable the response of the Nodal signal locally in this tissue. In contrast, there may be regionalized activity of receptors and agonists that enable the response to the Nodal signal in the LPM. However, this remains a conjecture as the expression of all components including Nodal receptors has not been examined exhaustively.

\section{Outstanding issues}

We now have gleaned a fair understanding of the construction and the putative mode of action of the LR organizer of the mouse embryo, the likely nature of the positional cues and some of the molecular determinants for transducing the organizer signal to the effector tissue.
There are, however, several burning questions that demand further clarification. They include the following: (a) how is the planar polarity of the pit cells translated to the positioning and/or the chirality of cilia motion? (b) what is the mode of action of Cerl2 and other factors that modulate the Nodal activity differently on contralateral sides of the organizer? (c) what, how and by which route is the LR patterning cue transferred to the LPM? and (d) how may the response to the LR patterning signal be elicited specifically in the LPM and not elsewhere in the embryo?

\section{Abbreviations}

$\mathrm{AP}$, anterior-posterior; DV, dorso-ventral; LPM, lateral plate mesoderm; LR, left-right; NVP, nodal vesicular parcel; pkd 2, polycystic kidney disease 2 .

\section{Disclosures}

The authors declare that they have no disclosures.

\section{Acknowledgments}

The work in the Hamada laboratory was supported by grants from CREST (Core Research for Evolutional Science and Technology) of the Japan Science and Technology Corporation and the Ministry of Education, Culture, Sports, Science and Technology of Japan. Patrick P. L. Tam is a Senior Principal Research Fellow of the National Health and Medical Research Council of Australia (NHMRC grant 1003100).

\section{References}

I. Gardner RL: Normal bias in the direction of fetal rotation depends on blastomere composition during early cleavage in the mouse. PLoS ONE 2010, 5:e9610.

\section{FlOOOPrime} RECOMMENDED

2. Blum M, Andre P, Muders K, Schweickert A, Fischer A, Bitzer E, Bogusch S, Beyer T, van Straaten HW, Henny WM, Viebahn C: Ciliation and gene expression distinguish between node and posterior notochord in the mammalian embryo. Differentiation 2007, 75:133-46

FlOOOPrime
RECOMMENDED

3. Lee JD, Anderson KV: Morphogenesis of the node and notochord: the cellular basis for the establishment and maintenance of left-right asymmetry in the mouse. Dev Dyn 2008, 237:3464-76.

4. Concepcion D, Papaioannou VE: Nature and extent of left/right axis defects in $\mathbf{T}(\mathrm{Wis}) / \mathrm{T}(\mathrm{Wis})$ mutant mouse embryos. $\mathrm{Dev}$ Dyn 2014, 243:1046-53.

FlOOOPrime RECOMMENDED

5. Yamanaka Y, Tamplin OJ, Beckers A, Gossler A, Rossant J: Live imaging and genetic analysis of mouse notochord formation reveals regional morphogenetic mechanisms. Dev Cell 2007, 1 3:884-96. 
6. Sutherland MJ, Wang S, Quinn ME, Haaning A, Ware SM: Zic3 is required in the migrating primitive streak for node morphogenesis and left-right patterning. Hum Mol Genet 2013, 22:1913-23

\section{FlOOOPrime \\ RECOMMENDED}

7. Burtscher I, Lickert H: Foxa2 regulates polarity and epithelialization in the endoderm germ layer of the mouse embryo. Development 2009, I36:1029-38.

\section{FIOOOPrime}

8. Lopes SS, Lourenço R, Pacheco L, Moreno N, Kreiling J, Saúde L: Notch signalling regulates left-right asymmetry through ciliary length control. Development 2010, 137:3625-32.

\section{FlOOOPrime}

9. Conlon FL, Lyons KM, Takaesu N, Barth KS, Kispert A, Herrmann B, Robertson E]: A primary requirement for nodal in the formation and maintenance of the primitive streak in the mouse. Development 1994, 120:1919-28.

10. Hoodless PA, Pye M, Chazaud C, Labbé E, Attisano L, Rossant J, Wrana JL: FoxHI (Fast) functions to specify the anterior primitive streak in the mouse. Genes Dev 200I, I5:|257-7I.

II. Pulina M, Liang D, Astrof S: Shape and position of the node and notochord along the bilateral plane of symmetry are regulated by cell-extracellular matrix interactions. Biol Open 2014, 3:583-90.

\section{FlOOOPrime}

\section{RECOMMENDED}

12. Choksi SP, Lauter G, Swoboda P, Roy S: Switching on cilia: transcriptional networks regulating ciliogenesis. Development 20|4, |4|:|427-4|.

\section{FlOOOPrime}

\section{RECOMMENDED}

13. Kinzel D, Boldt K, Davis EE, Burtscher I, Trümbach D, Diplas B, AttiéBitach T, Wurst W, Katsanis N, Ueffing M, Lickert H: Pitchfork regulates primary cilia disassembly and left-right asymmetry. Dev Cell 2010, 19:66-77.

\section{FlOOOPrime}

14. Hashimoto M, Shinohara K, Wang J, Ikeuchi S, Yoshiba S, Meno C, Nonaka S, Takada S, Hatta K, Wynshaw-Boris A, Hamada H: Planar polarization of node cells determines the rotational axis of node cilia. Nat Cell Biol 20I0, I2:170-6.

\section{FlOOOPrime} RECOMMENDED

15. Yoshiba S, Hamada $\mathrm{H}$ : Roles of cilia, fluid flow, and $\mathrm{Ca}^{2+}$ signaling in breaking of left-right symmetry. Trends Genet 2014, 30:10-7.

16. Song H, Hu J, Chen W, Elliott G, Andre P, Gao B, Yang Y: Planar cell polarity breaks bilateral symmetry by controlling ciliary positioning. Nature 2010, 466:378-82.

\section{FlOOOPrime} RECOMMENDED

17. Alten L, Schuster-Gossler K, Beckers A, Groos S, Ulmer B, Hegermann J, Ochs M, Gossler A: Differential regulation of node formation, nodal ciliogenesis and cilia positioning by Noto and Foxj I. Development 2012, I39:| 276-84.

\section{FlOOOPrime}

\section{RECOMMENDED}

18. Blum M, Feistel $K$, Thumberger $T$, Schweickert $A$ : The evolution and conservation of left-right patterning mechanisms. Development 2014, I4I:1603-13.

19. Sampaio P, Ferreira RR, Guerrero A, Pintado P, Tavares B, Amaro J, Smith AA, Montenegro-Johnson T, Smith DJ, Lopes SS: Left-right organizer flow dynamics: how much cilia activity reliably yields laterality? Dev Cell 2014, 29:716-28.

20. Shinohara K, Kawasumi A, Takamatsu A, Yoshiba S, Botilde $Y$, Motoyama N, Reith W, Durand B, Shiratori H, Hamada H: Two rotating cilia in the node cavity are sufficient to break leftright symmetry in the mouse embryo. Nat Commun 20I2, 3:622.

\section{FlOOOPrime} RECOMMENDED

21. Yoshiba S, Shiratori H, Kuo IY, Kawasumi A, Shinohara K, Nonaka S, Asai Y, Sasaki G, Belo JA, Sasaki H, Nakai J, Dworniczak B, Ehrlich BE, Pennekamp P, Hamada $\mathrm{H}$ : Cilia at the node of mouse embryos sense fluid flow for left-right determination via Pkd2. Science 2012, 338:226-31.

\section{FlOOOPrime}

\section{RECOMMENDED}

22. Kamura K, Kobayashi D, Uehara Y, Koshida S, lijima N, Kudo A Yokoyama T, Takeda H: Pkd III complexes with Pkd2 on motile cilia and functions to establish the left-right axis. Development 20II, I38:1121-9.

\section{FloOPrime} RECOMMENDED

23. Field S, Riley K, Grimes DT, Hilton H, Simon M, Powles-Glover N, Siggers P, Bogani D, Greenfield A, Norris DP: Pkd III establishes left-right asymmetry and physically interacts with Pkd2. Development 2011, 138:1131-42.

\section{FlOOOPrime}

24. Gros J, Feistel K, Viebahn C, Blum M, Tabin CJ: Cell movements at Hensen's node establish left/right asymmetric gene expression in the chick. Science 2009, 324:94I-4.

25. Mendes RV, Martins GG, Cristovão AM, Saúde L: N-cadherin locks left-right asymmetry by ending the leftward movement of Hensen's node cells. Dev Cell 2014, 30:353-60.

\section{FlOOOPrime}

26. Nonaka S, Shiratori H, Saijoh $Y$, Hamada H: Determination of leftright patterning of the mouse embryo by artificial nodal flow. Nature 2002, 418:96-9.

\section{FlOOOPrime}

RECOMMENDED

27. Tanaka $Y$, Okada $Y$, Hirokawa N: FGF-induced vesicular release of Sonic hedgehog and retinoic acid in leftward nodal flow is critical for left-right determination. Nature 2005, 435:172-7.

\section{FlOOOPrime}

\section{RECOMMENDED}

28. Marques S, Borges AC, Silva AC, Freitas S, Cordenonsi M, Belo JA The activity of the Nodal antagonist Cerl-2 in the mouse node is required for correct L/R body axis. Genes Dev 2004, 18:2342-7.

\section{FlOOOPrime} RECOMMENDED

29. Schweickert A, Vick P, Getwan M, Weber T, Schneider I, Eberhardt M, Beyer T, Pachur A, Blum M: The nodal inhibitor Coco is a critical target of leftward flow in Xenopus. Curr Biol $2010,20: 738-43$

30. Nakamura T, Saito D, Kawasumi A, Shinohara K, Asai Y, Takaoka K, Dong F, Takamatsu A, Belo JA, Mochizuki A, Hamada H: Fluid flow and interlinked feedback loops establish left-right asymmetric decay of Cerl2 mRNA. Nat Commun 2012, 3:1322.

31. Inácio JM, Marques S, Nakamura T, Shinohara K, Meno C, Hamada $\mathrm{H}$ Belo JA: The dynamic right-to-left translocation of Cerl2 is involved in the regulation and termination of Nodal activity in the mouse node. PLOS ONE 2013, 8:e60406.

32. Shiratori $\mathrm{H}$, Hamada $\mathrm{H}$ : The left-right axis in the mouse: from origin to morphology. Development 2006, 133:2095-104. 
33. Oki S, Hashimoto R, Okui Y, Shen MM, Mekada E, Otani H, Saijoh Y, Hamada $H$ : Sulfated glycosaminoglycans are necessary for Nodal signal transmission from the node to the left lateral plate in the mouse embryo. Development 2007, 134:3893-904.

34. Furtado MB, Solloway MJ, Jones VJ, Costa MW, Biben C, Wolstein O, Preis JI, Sparrow DB, Saga Y, Dunwoodie SL, Robertson EJ, Tam PPL, Harvey RP: BMP/SMADI signaling sets a threshold for the left/ right pathway in lateral plate mesoderm and limits availability of SMAD4. Genes Dev 2008, 22:3037-49.

35. Saijoh Y, Viotti M, Hadjantonakis A: Follow your gut: relaying information from the site of left-right symmetry breaking in the mouse. Genesis 20I4, 52:503-14.

36. Saund RS, Kanai-Azuma M, Kanai Y, Kim I, Lucero MT, Saijoh Y: Gut endoderm is involved in the transfer of left-right asymmetry from the node to the lateral plate mesoderm in the mouse embryo. Development 2012, I39:2426-35.

37. Viotti M, Niu L, Shi S, Hadjantonakis A: Role of the gut endoderm in relaying left-right patterning in mice. PLoS Biol 2012, 10: el00I276
38. Norris DP: Cilia, calcium and the basis of left-right asymmetry. BMC Biol 2012, 10:102.

FIOOOPrime

39. Kanai-Azuma M, Kanai Y, Gad JM, Tajima Y, Taya C, Kurohmaru M, Sanai Y, Yonekawa H, Yazaki K, Tam PPL, Hayashi Y: Depletion of definitive gut endoderm in Sox 17-null mutant mice. Development 2002, I 29:2367-79.

40. Fuller K, O'Connell JT, Gordon J, Mauti O, Eggenschwiler J: Rab23 regulates Nodal signaling in vertebrate left-right patterning independently of the Hedgehog pathway. Dev Biol 2014, 391:182-95.

\section{FlOOOPrime}

RECOMMENDED

4I. Müller P, Rogers KW, Jordan BM, Lee JS, Robson D, Ramanathan S, Schier AF: Differential diffusivity of Nodal and Lefty underlies a reaction-diffusion patterning system. Science 20I2, 336:72I-4.

FlOOOPrime

RECOMMENDED 